\title{
Functional correlation between breathing and emotional states
}

\begin{abstract}
Breathing changes in response to emotional states, such as sadness, happiness, anxiety or fear. On the other hand, emotional states change the pattern, rate and depth of breathing. A complex coordination, not yet fully understood, involving widely dispersed brain centres in the cerebral cortex, limbic system, medullary and pontine areas, together control the correlation between breathing and emotion. This intricate correlation between breathing and emotion is essential to synchronize metabolism, energetic and other physiological parameters of homeostasis with changes in the environment. Adept correlation of breathing with emotional states not only maintains homeostasis but is also essential for survival.
\end{abstract}

Volume 3 Issue 5 - 2017

\author{
Anjali A Sarkar \\ Freelance, USA
}

Correspondence: Anjali A Sarkar, Freelance, 2916 Weisman Road Silver Spring MD 20902, USA, Email sarkaraa@gmail.com

Received: March 14, 2017| Published: May 18, 2017

\section{Introduction}

Almost everyone is intuitively aware of a connection between breathing and emotion. We know that when we are agitated and anxious our breath is rapid and shallow and when we're relaxed and content our breath is slow and deep. We might be somewhat less aware, however that when we compel our breath to be rapid and shallow we become agitated and anxious and when we compel our breath to be slow and deep we promote relaxation and contentment. Breathing is the only essential physiological function that is both under voluntary and involuntary control. For example, the heart needs to beat for us to live but we cannot increase or decrease our heart rate at will. Breathing on the other hand is more amenable to conscious regulation. e

Three areas of the brain regulate breathing and respiration. Here, breathing is defined as the physiological process of inhalation and exhalation that carries oxygen laden air into the alveoli of the lungs and expels carbon dioxide laden air into the atmosphere, whereas respiration is defined as the cellular metabolism process of oxygen transfer and the combustion of high energy bio molecules that maintain homeostasis. Widely dispersed centres located in the brainstem, the limbic system and the cerebral cortex regulate breathing and respiration. The regulation of breath by the brain stem is largely unconscious and serves to maintain homeostasis whereas the regulation of breath by the limbic system generates emotional repercussions, while the higher cognitive centers of the cerebral cortex exert voluntary and intentional control on breathing.

\section{Effect of emotion on breathing}

Neuroscientists have been using braining imaging studies to understand anatomical signatures for emotional states, ${ }^{1-6}$ establishing the brain regions that are activated in synchrony in a given emotion or mood. Emotions generate not only brain signatures but also body (somatic) signatures in the form of responses in the peripheral nervous system and behavioral expression. Emotion changes heart rate, blood pressure, skin conductance and breathing, ${ }^{7}$ largely unconsciously.

Animal model studies have revealed detailed mechanisms underlying the neural control of breathing and the coordination of emotion and breathing. For example, sighs, that are long deep breaths and express sadness, relief or exhaustion are regulated by a peptidergic circuit regulated by bombesin-like neuropeptide genes neuromedin
$\mathrm{B}(\mathrm{Nmb})$ or gastrin releasing peptide (Grp) expressed in neurons of a breath control center (RTN/pFRG) that project to the respiratory rhythm generator (preBötzinger Complex). ${ }^{8}$

\section{Effect of breathing on emotion}

Conscious regulation of the breath activates the motor cortex. ${ }^{9}$ Observation of the breath has revealed that breath cycles through a predominance of the right and left nostril with brief intervening periods where the breathing in both nostrils are equally balanced. Yogic practice of alternate nostril breathing (Nadi Shodhan Pranayama, translated as the cleansing of the nerve centres) has long espoused a calming effect when breathing is restricted to the left nostril and an activating or energizing effect when breathing is restricted to the right nostril. In a single breathing cycle, extension of the inhalation phase has an energizing and activating effect whereas the extension of the exhalation phase has a relaxing and calming effect.

Respiratory symptoms are the best predictor of panic attacks, as defined in the DSM IV. Studies have shown that particularly in a carbon dioxide enriched environment, respiration is highly correlated to feelings of fear and discomfort. ${ }^{10}$

\section{Therapeutic applications of breath-emotion dynamics}

Symptoms and treatment of cancer generate extreme anxiety. A study conducted on 16 age-matched cancer patients receiving chemotherapy, shows the beneficial effect of four pranayama breathing techniques on cancer related emotional symptoms. ${ }^{11}$ Simple breathing techniques feasible to be practiced in class and at home by cancer patients is used in this study. The first technique involves maintaining a continuous awareness of the breath; the second technique (Ujjayi Pranayama, translated as the victorious breath) involves a partially closed glottis, deep inhalations extending the lower and upper lungs and extended exhalation; the third technique (Kapalabhati Pranayama) involves gentle inhalation, brief retention and forceful exhalation, tailored to the strength of the patient; the fourth technique (NadiShodhan Pranayama) involved using the fingers in a specific orientation (mudra) to close alternate nostrils so as to restrict breath to either the right or left nostril. The practice of these four breathing techniques significantly reduced fatigue, sleep disturbance, anxiety, depression and stress in the limited sample of cancer patients.

A larger study on 74 young adults that involves training in Ujjayi 
breathing, Bhastrika pranayama and Sudarshan Kriya for 20hours over a five day period shows improvements in self-reported depression, stress, satisfaction, social connectedness and gratitude. ${ }^{12}$ Breathing based meditation techniques have also been shown to reduce post traumatic stress disorder in US army veterans ${ }^{13}$ and effectively reduce anxiety and depression in COPD patient. ${ }^{14}$

\section{Conclusion}

Before adopting any Yoga-based breathing technique, it is crucial to ascertain the skill, experience and knowledge of the instructor. This is because just as correct breath control technique can alleviate undesirable emotional states, incorrect breathing techniques can be harmful, generating neural injury in the brain that can affect a wide range of physiological systems. This is supported by studies on patients with abnormal breathing patterns during sleep that show injury in the medulla, pons, cerebellum, neurotransmission through serotonergic, adrenergic and peptidergic neurons, somatosensory and limbic cortices and the hippocampus. ${ }^{15}$

\section{Acknowledgements}

None.

\section{Conflict of interest}

Author declares that there is no conflict of interest.

\section{References}

1. Contreras Rodriguez O, Pujol J, Batalla I, et al. Disrupted neural processing of emotional faces in psychopathy. Soc Cogn Affect Neurosci. 2014;9(4):505-512.

2. Pujol J, Giménez M, Ortiz H, et al. Neural response to the observable self in social anxiety disorder. Psychol Med. 2013;43(4):721-731.

3. Tettamanti M, Rognoni E, Cafiero R, et al. Distinct pathways of neural coupling for different basic emotions. Neuroimage. 2012;59(2):1804-1817.

4. MacConnell G, Aston M, Randel P, et al. Nurses' experiences providing bereavement follow-up: an exploratory study using feminist poststructuralism. J Clin Nurs. 2013;22(7-8):1094-1102.
5. van der Zwaag MD, Dijksterhuis C, de Waard D, et al. The influence of music on mood and performance while driving. Ergonomics. 2012;55(1):12-22.

6. van der Zwaag W, Da Costa SE, Zurcher NR, et al. A 7 tesla FMRI study of amygdala responses to fearful faces. Brain Topogr. 2012;25(2):125-128.

7. Boiten FA, Frijda NH, Wientjes CJ. Emotions and respiratory patterns: review and critical analysis. Int $J$ Psychophysiol. 1994;17(2):103-128.

8. Li P, Janczewski WA, Yackle K, et al. The peptidergic control circuit for sighing. Nature. 2016;530(7590):293-297.

9. Masaoka Y, Izumizaki M, Homma I. Where is the rhythm generator for emotional breathing? Prog Brain Res. 2014;209:367-377.

10. Colasanti A, Salamon E, Schruers K, et al. Carbon dioxideinduced emotion and respiratory symptoms in healthy volunteers. Neuropsychopharmacology. 2008;33(13):3103-3110.

11. Dhruva A, Miaskowski C, Abrams D, et al. Yoga breathing for cancer chemotherapy-associated symptoms and quality of life: results of a pilot randomized controlled trial. J Altern Complement Med. 2012;18(5):473-479.

12. Goldstein MR, Lewis GF, Newman R, et al. Improvements in wellbeing and vagal tone following a yogic breathing-based life skills workshop in young adults: Two open-trial pilot studies. Int $J$ Yoga. 2016;9(1):20-26.

13. Seppala EM, Nitschke JB, Tudorascu DL, et al. Breathing-based meditation decreases posttraumatic stress disorder symptoms in U.S. military veterans: a randomized controlled longitudinal study. $J$ Trauma Stress. 2014;27(4):397-405.

14. Valenza MC, Valenza Peña G, Torres Sánchez I, et al. Effectiveness of controlled breathing techniques on anxiety and depression in hospitalized patients with COPD: a randomized clinical Trial. Respir Care. 2014;59(2):209-215.

15. Harper RM, Kumar R, Ogren JA, et al. Sleep-disordered breathing: effects on brain structure and function. Respir Physiol Neurobiol. 2013;188(3):383-391. 